
\title{
3 Research S Suare \\ Irritable Bowel Syndrome and Migraine: Evidence From Mendelian Randomization Analysis in the UK Biobank
}

Jie Chen ( $\square$ med_chenjie@zju.edu.cn )

Zhejiang University https://orcid.org/0000-0002-4029-4192

\section{Xuejie Chen}

Department of Gastroenterology, The third Xiangya Hospital, Central South University

\section{Ying Xie}

Centre for global health, Zhejiang University School of Medicine

\section{Yuhao Sun}

Centre for Global Health, Zhejiang University School of Medcine

\section{Xiaoyan Wang}

Department of Gastroenterology, The third Xiangya Hospital, Central South University

Therese Hesketh

Centre for Global Health, Zhejiang University School of Medicine; Institute for Global Health, University College London

\section{Research article}

Keywords: Migraine, Irritable bowel syndrome, Mendelian randomization, Epidemiology, UK Biobank

Posted Date: April 8th, 2021

DOl: https://doi.org/10.21203/rs.3.rs-401295/v1

License: (9) This work is licensed under a Creative Commons Attribution 4.0 International License. Read Full License 


\section{Abstract}

Background: IBS and Migraine are two diseases featuring high prevalence. Previous studies have suggested a relationship between Irritable Bowel Syndrome (IBS) and migraine, although the causal association remains unclear. We sought to explore the causal association between IBS and migraine, and to prove the importance of migraine prevention in IBS patients.

Methods: This study used a two-sample Mendelian-randomization analysis to explore the association of IBS with migraine. Genetic association with migraine were acquired from the UK Biobank (UKB) genetic databases (cases: 1,072; controls: 360,122). We performed estimation using Inverse Variance Weighting (IVW), along with Maximum Likelihood, MR-RAPS, MR-Egger and Weighted Median for sensitivity analysis. Considering possible bias, we also conducted polymorphism, heterogeneity, and directional analysis.

Results: The IVW estimation genetically predicted the causal association between IBS and migraine (OR=1.09, $95 \% \mathrm{Cl} 1.01$ to $1.17, p=0.03$ ). Neither statistical horizontal pleiotropy (MR Egger $p=0.42$; MRPRESSO $p=0.78$ ) nor possible heterogeneity (IVW Q $=26.15, p=0.80$ ) was found. Reverse causation was also not detected ( $p$ steiger $<0.01)$.

Conclusion: Mendelian randomization analysis supported a positive-going causal association of IBS with migraine, providing enlightenment for disease prevention and control.

\section{Introduction}

Irritable bowel syndrome (IBS) is a common chronic gastrointestinal disorder with a pooled prevalence of $11.2 \%$ worldwide [1]. Its typical symptoms include abdominal pain and abnormal bowel motility without organic lesion [2]. A prevalence cohort study suggested that IBS patients were more likely to have migraine, fibromyalgia, and depression, known as the functional somatic syndromes. Migraine is also a chronic disorder, affecting approximately $6 \%$ of men and 15 to $17 \%$ of women $[3,4]$. It features recurrent headache disorder which may be accompanied by various autonomic, affective, cognitive, and sensory symptoms [5]. For young women, migraine is also ranked top in the cause of disability-adjusted life years (DALYs) [6].

IBS and migraine have posed threat to a large population, affecting their daily life activities [7]. According to reports, both IBS and migraine damage life quality $[1,8]$, which may exert a burden on patients and the health care system. As an important public health problem [9], migraine is even related to a high level of disability [8]. Thus, figuring out the relationship of the two concomitant diseases may pose a vital significance to public health.

Previous studies have observed the association of IBS and migraine [10]. Among IBS patients, approximately $25-50 \%$ had migraine or headache compared with only $4-19 \%$ among the controls [11]. The Odds Ratio of IBS patients co-existing a migraine or headache reached 2.66 [12]. However, most of 
the above studies are observational studies and the causal relationship between IBS and migraine is still controversial. Although evidence has indicated that symptoms of the central nervous system (CNS) may occur following the gastrointestinal dysfunction [13], we could not exclude the reverse causality and existing confounding factors.

Mendelian randomization (MR) analysis is a gene-based approach that can estimate the causal relationship between an exposure (such as IBS) and an outcome (for example, migraine). Genetic variants are used as the instrumental variables (IVs) of exposure [14]. The individual genetic variants were pooled for population effect estimation, similar to a random effects meta-analysis. On the one hand, gametes are formed according to the Mendelian genetic law that parental alleles are randomly assigned to offspring. Therefore, MR can avoid confounding factors such as environmental exposure, socioeconomic status and behavior. On the other hand, since the genetic variation comes from the parents and remains unchanged after birth, the association between genetic variation and outcome is chronological, overcoming the problem of reverse causation.

We hypothesized that migraine may be the downstream effect of IBS. We performed two-sample MR (TSMR) to infer the causal relationship with no need for individual-level data. We chose single nucleotide polymorphisms (SNPs) from Genome-Wide Association Studies (GWAS) to assess the association of IBS and migraine.

\section{Methods}

\section{Mendelian randomization and assumptions}

This analysis proceeds from summary data without individual level resources. We conducted two-sample MR using genetic variants as IVs. The SNPs can be considered as valid IVs for the IBS if they satisfied the three core assumptions of MR: (i) genetic variants as IVs are strongly associated with exposures (Correlation hypothesis); (ii) genetic variants are independent of the known or unknown confounders (Independence hypothesis); (iii) genetic variants only associate with outcomes through the exposure (Exclusion hypothesis).

\section{Data resources}

The SNPs associated with IBS were selected from the published open GWAS database up to date, including 1,121 cases and 360,073 population controls [15]. The SNPs associated with migraine were obtained from the UK Biobank (UKB) genetic data (1,072 cases and 360,122 controls). The UKB project is known as a prospective cohort study, recruiting participants ranging from 40 to 69 years-old in the United Kingdom [16]. It collected individual genotypes as well as health-related information. UK Biobank has already got ethical approval (REC reference for UK Biobank is 11/NW/0382). To improve accuracy, our study chose patients with the main diagnosis in hospital as cases according to the International Classification of Diseases version-10 (recorded as "Diagnoses - main ICD10" in UKB), while other individuals as controls. 


\section{SNP Selection}

Aiming to meet the three core assumptions, standards were set for valid SNPs. We used SNPs at a linkage disequilibrium (LD) threshold $\mathrm{R}^{2}<0.001$, with a certain mutation frequency (Minor Allele Frequency (MAF) $\geq 5 \%)$ and predicted the exposure significantly at the genetic level $(p<5 \times 10-8)$. Since no eligible SNPs were dug out, we used a higher cut-off $(p<1 \times 10-5)$ to obtain SNPs related to the exposure. For harmonization, the palindromic SNPs were also excluded to avoid that the direction of the positive and negative chains cannot be determined for the same alleles on both strands.

\section{Main statistical analysis}

We performed Inverse Variance Weighted (IVW) as the main analysis, which is a weighted estimation using the individual ratio from meta-analysis literature. IVW estimate is consistent and efficient if the pleiotropic effects of IVs are zero. Additionally, with a larger sample size, the estimation will be closer to the true value [17].

\section{Sensitivity analyses}

Except for IVW, we also conduct other methods to test the conformity of test results. Maximum Likelihood $(\mathrm{ML})$ is a traditional means with low standard error. It estimates the probability distribution parameters by maximizing the likelihood function. Although it may be biased with limited sample sizes, the bias is so small that can be ignored biologically [18]. Robust analysis method refers to the means that can indicate the causal relationship even under a weak assumption due to pleiotropy or linkage disequilibrium. To provide a more credible estimate, we used robust analyses like MR-Egger, Weighted Median and MRPRESSO. Originated from assessing the publication bias [19], MR-Egger estimates the effect of geneoutcome on gene-exposure using weighted linear regression. It allows the inclusion of the pleiotropic genetic variants if conform with the InSIDE hypothesis that the pleiotropic effect is independent of the association between IVs and exposure. MR-Egger can assess the pleiotropic effects using the intercept [20]. However, MR-Egger analysis is prone to be affected by outliers, resulting in relatively imprecise and low power [21]. The Weighted Median (WM) can improve the accuracy of the simple median estimator by including the weight of the ordered ratio. WM can provide a valid and consistent estimate when more than $50 \%$ of the involved information comes from valid IVs [17]. MR-PRESSO is a variant of IVW, excluding genetic variants whose causal estimation is different from other IVs significantly. The MRPRESSO method can provide valid results if the horizontal pleiotropy exists in less than $50 \%$ of the IVs [22]. The Robust Adjusted Profile Score (MR-RAPS) features high statistical power, which is robust when systematic pleiotropy exists and unbiases with weak instruments. Considering the measurement error in the association of SNPs and exposure, MR-RAPS can reduce horizontal pleiotropy [23].

To test the conformity of each SNP, we also performed a Leave-one-out analysis by conducting the Mendelian randomization leaving each genetic variant one by one. If the causal relationship is still significant statistically after excluding the none-specific SNP, it provides more credible evidence for the association. Pleiotropy means a genetic variation is associated with multiple risk factors, including 
horizontal pleiotropy and vertical pleiotropy. Since vertical pleiotropy neither violates the core hypothesis of Mendelian randomization nor causes any bias, we only consider horizontal pleiotropy, which may bias the independence hypothesis and the exclusivity hypothesis. We used MR-Egger intercept and MRPRESSO analysis to verify and correct for horizontal pleiotropy. As for heterogeneity, Cochran Q statistics were calculated to examine the heterogeneity among different genetic variations. We also drew the funnel plot for visual examination. MR-Steiger can test the confidence of the causal direction of IBS and migraine using summary data [24]. It was also conducted to identify a cause from a downstream effect. The results of both MR analyses and sensitivity estimates were considered statistically significant at $p<$ 0.05 , with two-tailed testing. All estimations were conducted in $\mathrm{R}$ Version 4.0.3 with $\mathrm{R}$ package "TwoSampleMR" and "MRPRESSO". We used summary data publicly available which need no ethical approval.

\section{Results}

According to the selection criteria, we first chose 42 SNPs that were significantly related to IBS $(p<1 \times 10$ $5)$ independently $\left(R^{2}<0.001\right)$. F-statistics ranged from 19.54 to 28.67 , indicating the weak instrumental bias can be ignored statistically (Details in Supplementary Tables S1). Given the high comorbidity of IBS and migraine, possible confounders were also considered, such as SNPs related to fibromyalgia, chronic fatigue syndrome and depression. We then excluded 8 SNPs for being palindromic (rs10910535, rs12186442, rs138082719, rs17677265, rs201655992, rs56135098, rs79333841, rs9367125). Finally, a total of 34 SNPs were selected (Supplementary Tables S1).

The main analysis IVW suggested a causal association of IBS and migraine $(\mathrm{OR}=1.09,95 \% \mathrm{Cl} 1.01$ to $1.17, p=0.03)$. For sensitivity analysis, we also conducted MR-Egger, ML, WM, and MR-RAPS to confirm the conformity of test results. (Figs. 2) The directions of the five models were consistent, ML and MRRAPS were significant, while MR-Egger and WM were not significant statistically. As for the SNP conformity, we performed a Leave-one-out analysis and drew a forest map. The forest map intuitively showed a stable result (Supplementary Figures S3).

Horizontal polymorphism and heterogeneity analyses were performed as well for sensitivity quality control. MR-Egger intercept and MR-PRESSO analysis identified no horizontal pleiotropy statistically (MR Egger $p=0.42$; MR-PRESSO $p=0.78$ ). Since there was no statistical horizontal pleiotropy, we used a fixedeffect model. To test the heterogeneity, we calculated the Cochran Q statistics, find no heterogeneity statistically between IV estimates (IVW Q $=26.15, p=0.80$ ). The funnel plot also indicated the unlikely heterogeneity among SNPs (Supplementary Figures S2). These results suggested the powerful estimate and weak bias in the MR analysis. Besides, MR-Steiger indicated IBS as the cause while migraine as the downstream consequence ( $p$ steiger $<0.01, p M R<0.05$ ).

\section{Discussion}


To the best of our knowledge, it is the first study illustrating the causal association between IBS and migraine from the genetic perspective.

The causal association supported the possible common pathogenesis or associated pathways between the two diseases. Former researchers have proposed several hypotheses which may explain the mechanisms. The leaky gut hypothesis indicated that altered permeability and impaired barrier of the gastrointestinal tract (GIT) may lead to the leakage. Bacterial by-products may go across the intestinal mucosa and enter the bloodstream, thus causing migraine $[25,26]$. In addition, the Gut-brain axis is an acknowledged theory, pointing out the bidirectional relationship between the gastrointestinal system and central nervous system (CNS). The CNS affects GIT by the hypothalamic-pituitary-adrenal (HPA) axis, the sympathetic and parasympathetic branches. In turn, the gastrointestinal system is also indicated to influence the CNS, including emotional behavior, pain-modulation systems, and brain neurotransmitter systems $[27,28]$. The causal relationship of IBS and migraine may also relate to the GIT-CNS pathways mediated by the Gut-brain axis. Additionally, the intestinal microbe is believed to affect neurotransmitter levels, such as serotonin [29]. Although, only $3 \%$ of the serotonin locate in the CNS, it may cause nervous system dysfunction or disorders like a migraine. While most exist in the GIT, where it can be released from enterochromaffin cells and regulated by Enteric bacteria. Thus, a therapy combining restore intestinal function such as probiotics may benefit migraine patients [26]. Therapy regulating serotonin receptors was reported working for both IBS and migraine [30].

Among the five MR methods, IVW, ML and MR-RAPS revealed the causal relationship of IBS and migraine while MR-Egger and WM showed no significance statistically. Considering the pleiotropic effects of the same confounder, MR-Egger has greater error rates than IVW [21]. Since the low statistical power, we stress more importance to the consistency of the direction of the slope rather than the significance [31]. Given that the five methods were in the same direction, we conclude that IBS was causally associated with migraine.

Clarifying the causal relationship and exploring the mechanism between the two diseases are conducive to improve prevention and control. On the one hand, IBS patients should attach importance to the prevention of migraine. Though frustrating, IBS does not pose a serious threat to a targeted organ, like cancers [32]. A nationwide cohort study in Denmark even suggested that IBS patients had less risk of colorectal cancers with a standardized incidence ratio (SIR) of 0.67 [33]. In comparison, migraine may account for more serious consequences and burdens. The severe pain may lead to a higher risk of suicide attempt [34], cardiovascular disease events [35] and stroke [36]. Thus, it is important to prevent migraine among IBS patients from the first beginning. Preventive therapy has been suggested when indications occurred, like more than four headaches or at least eight days of headache a month, debilitating headaches, etc. Medication, as well as relaxation training, thermal biofeedback and cognitive behavior therapy, was also suggested for the prevention of migraine [37]. On the other hand, given the co-exist of the two diseases, new treatments should be developed accordingly. Food allergies (FA) were reported both related to IBS relapse and migraine initiation [10]. Excessive specific IgGs in food may cause FA. A previous randomized controlled trial indicated that an IgG-based elimination diet can reduce IBS and 
migraine symptoms [38]. Melatonin, a natural hormone in the body maintaining the biological clock was also found to reduce pains of IBS and migraine [39]. A randomized controlled trial showed the improvement of the symptoms among IBS patients, suggesting a peripheral anti-5-HT-like effect of melatonin [40]. A clinical trial also reported melatonin can decrease the frequency, duration and intensity of a migraine attack [41]. Moreover, neurokinin-1 (NK1) receptor antagonists also play a role in relieving the symptoms of IBS and migraine, as the receptor was found both in CNS and GIT [42]. Further pathogenesis investigation and new-drug development would be valuable in controlling the two diseases.

Admittedly, though we testified the causal association between IBS and migraine, evidence from both preclinical medicine and clinical practice are still needed to explore molecular mechanism and therapies. On the other hand, despite the MR analysis was less likely to be affected by confounders compared with other observational designs, limitations still exist. First, we used a higher cut-off $(p<1 \mathrm{e}-5)$ to obtain more SNPs, which may include weak instrumental variables, reducing the effectiveness. However, the Fstatistics we calculated varied from 19.54 to 28.67 , suggesting the effect of weak IVs was not substantial. Second, MR analysis may underestimate biological effects while overestimating genetic associations, known as the "Beavis Effect". Concerning the potential association between SNPs and confounding factors, we excluded the weak SNPs using F-statistic and check several reported confounders, excluding related SNPs. Third, it is hard to satisfy the "Exclusion hypothesis" entirely, limiting the association of SNPs to the outcome only through exposure. To detect the bias caused by horizontal pleiotropy, we utilized the MR-Egger intercept and MR-PRESSO analysis, find a minimal pleiotropy effect. Also, we incorporated more IVs, avoiding specific SNPs playing a decisive role. Fourth, the SNPs we used were collected from the European populations, which may differ in other populations. Further analysis concerning other groups of people would provide more evidence.

\section{Conclusion}

In this study, we found that migraine may be the downstream consequence of IBS using Mendelian randomization analysis. It gives reminder to clinical practitioners that concerns and preventions for migraine should be taken when patients are diagnosed with IBS. Additionally, pathogenesis and treatments research concerning both of the two disease should be taken into actions.

\section{Abbreviations}

CNS: Central nervous system

FA: Food allergies

GIT: Gastrointestinal tract

GWAS: Genome-Wide Association Studies

HPA: Hypothalamic-pituitary-adrenal 
IBS: Irritable bowel syndrome

IVs: Instrumental variables

IVW: Inverse Variance Weighting

LD: Linkage disequilibrium

MAF: Minor Allele Frequency

ML: Maximum Likelihood

MR: Mendelian randomization

NK1: Neurokinin-1

RAPS: Robust Adjusted Profile Score

SIR: Standardized incidence ratio

SNPs: Single nucleotide polymorphisms

TSMR: Two-sample MR

UKB: the UK Biobank

WM: Weighted Median

\section{Declarations}

Ethics approval and consent to participate

Exempt from approval because it is no longer human subjects data

\section{Consent for publication}

Not applicable

\section{Availability of data and materials}

All data used for the analysis were derived from published GWAS.

Migraine data are available in https://gwas.mrcieu.ac.uk/datasets/ukb-d-G43/

IBS data are available in https://gwas.mrcieu.ac.uk/datasets/ukb-d-K58/ 
The authors have no relevant affiliations or financial involvement with any organization or entity with a financial interest or financial conflict in the manuscript.

\section{Funding}

National Natural Science Foundation of China (81970494);

Key Project of Research and Development Plan of Hunan Province(2019SK2041)

\section{Author contributions}

Author contributions: Jie Chen, Xuejie Chen and Ying Xie contributed data acquisition, analysis and writing. Yuhao Sun helped analyze the data. Xiaoyan Wang and Therese Hesketh contributed to project design and manuscript revision. All authors approved the final version of the manuscript.

\section{Acknowledgments}

We appreciate MRC Integrative Epidemiology Unit (https://gwas.mrcieu.ac.uk/ ) and Neale Lab (http://www.nealelab.is/uk-biobank ) for the publicly available summary data.

\section{References}

1. Enck P, Aziz Q, Barbara G, et al. Irritable bowel syndrome. Nat Rev Dis Primers, 2016,2:16014.

2. Kim Y S, Kim N. Sex-Gender Differences in Irritable Bowel Syndrome. J Neurogastroenterol Motil, 2018,24(4):544-558.

3. Stewart W F, Shechter A, Rasmussen B K. Migraine prevalence. A review of population-based studies. Neurology, 1994,44(6 Suppl 4): S17-S23.

4. Lipton R B, Stewart W F, Diamond S, et al. Prevalence and burden of migraine in the United States: data from the American Migraine Study II. Headache, 2001,41(7):646-657.

5. Burstein R, Noseda R, Borsook D. Migraine: multiple processes, complex pathophysiology. J Neurosci, 2015,35(17):6619-6629.

6. Steiner $T$ J, Stovner $L$ J, Jensen R, et al. Migraine remains second among the world's causes of disability, and first among young women: findings from GBD2019[J]. J Headache Pain, 2020,21(1):137.

7. Perveen I, Parvin R, Saha M, et al. Prevalence of Irritable Bowel Syndrome (IBS), Migraine and CoExisting IBS-Migraine in Medical Students. J Clin Diagn Res, 2016,10(11):C9-C13.

8. Takeshima T, Wan Q, Zhang Y, et al. Prevalence, burden, and clinical management of migraine in China, Japan, and South Korea: a comprehensive review of the literature. J Headache Pain, 2019,20(1):111.

9. Burch R, Rizzoli P, Loder E. The Prevalence and Impact of Migraine and Severe Headache in the United States: Figures and Trends From Government Health Studies. Headache, 2018,58(4):496-505. 
10. Georgescu D, Reisz D, Gurban C V, et al. Migraine in young females with irritable bowel syndrome: still a challenge. Neuropsychiatr Dis Treat, 2017,14:21-28.

11. Cole J A, Rothman K J, Cabral H J, et al. Migraine, fibromyalgia, and depression among people with IBS: a prevalence study. BMC Gastroenterol, 2006,6:26.

12. Chang F Y, Lu C L. Irritable bowel syndrome and migraine: bystanders or partners? J Neurogastroenterol Motil, 2013,19(3):301-311.

13. Rao M, Gershon M D. The bowel and beyond: the enteric nervous system in neurological disorders. Nat Rev Gastroenterol Hepatol, 2016,13(9):517-528.

14. Evans D M, Davey S G. Mendelian Randomization: New Applications in the Coming Age of Hypothesis-Free Causality. Annu Rev Genomics Hum Genet, 2015,16:327-350.

15. The MRC IEU OpenGWAS data infrastructure. Ben Elsworth, Matthew Lyon, Tessa Alexander, et al. bioRxiv 2020.08.10.244293v1.

16. Bycroft C, Freeman C, Petkova D, et al. The UK Biobank resource with deep phenotyping and genomic data. Nature, 2018,562(7726):203-209.

17. Bowden J, Davey S G, Haycock P C, et al. Consistent Estimation in Mendelian Randomization with Some Invalid Instruments Using a Weighted Median Estimator. Genet Epidemiol, 2016,40(4):304-314.

18. Milligan B G. Maximum-likelihood estimation of relatedness. Genetics, 2003,163(3):1153-1167.

19. Egger M, Davey SG, Schneider M, et al. Bias in meta-analysis detected by a simple, graphical test. BMJ, 1997; 315(7109): 629-634.

20. Burgess S, Davey S G, Davies N M, et al. Guidelines for performing Mendelian randomization investigations. Wellcome Open Res, 2019,4:186.

21. Burgess $S$, Thompson $S G$. Interpreting findings from Mendelian randomization using the MR-Egger method. Eur J Epidemiol, 2017,32(5):377-389.

22. Verbanck M, Chen C Y, Neale B, et al. Detection of widespread horizontal pleiotropy in causal relationships inferred from Mendelian randomization between complex traits and diseases. Nat Genet, 2018,50(5):693-698.

23. Zhao Q, Chen Y, Wang J, et al. Powerful three-sample genome-wide design and robust statistical inference in summary-data Mendelian randomization. Int J Epidemiol, 2019,48(5):1478-1492.

24. Hemani G, Tilling K, Davey S G. Orienting the causal relationship between imprecisely measured traits using GWAS summary data. PLoS Genet, 2017,13(11): e1007081.

25. Quigley E M. Leaky gut - concept or clinical entity? Curr Opin Gastroenterol, 2016,32(2):74-79.

26. Xie Y, Zhou G, Xu Y, et al. Effects of Diet Based on IgG Elimination Combined with Probiotics on Migraine Plus Irritable Bowel Syndrome. Pain Res Manag, 2019,2019:7890461.

27. Mayer E A, Tillisch K, Gupta A. Gut/brain axis and the microbiota. J Clin Invest, 2015,125(3):926-938.

28. Arzani M, Jahromi S R, Ghorbani Z, et al. Gut-brain Axis and migraine headache: a comprehensive review. J Headache Pain, 2020,21(1):15. 
29. O'Mahony S M, Clarke G, Borre Y E, et al. Serotonin, tryptophan metabolism and the brain-gutmicrobiome axis. Behav Brain Res, 2015,277:32-48.

30. Camilleri M. Review article: new receptor targets for medical therapy in irritable bowel syndrome. Aliment Pharmacol Ther, 2010,31(1):35-46.

31. Yeung C, Schooling C M. Systemic inflammatory regulators and risk of Alzheimer's disease: a bidirectional Mendelian-randomization study. Int J Epidemiol, 2020.

32. Malone M A. Irritable bowel syndrome. Prim Care, 2011,38(3):433-447.

33. Nørgaard M, Farkas D K, Pedersen L, et al. Irritable bowel syndrome and risk of colorectal cancer: a Danish nationwide cohort study. Br J Cancer, 2011,104(7):1202-1206.

34. Breslau N, Schultz L, Lipton R, et al. Migraine headaches and suicide attempt. Headache, 2012,52(5):723-731.

35. Kurth T, Winter A C, Eliassen A H, et al. Migraine and risk of cardiovascular disease in women: prospective cohort study. BMJ, 2016,353: i2610.

36. Øie L R, Kurth T, Gulati S, et al. Migraine and risk of stroke. J Neurol Neurosurg Psychiatry, 2020,91(6):593-604.

37. Ha H, Gonzalez A. Migraine Headache Prophylaxis. Am Fam Physician, 2019,99(1):17-24.

38. Aydinlar E I, Dikmen P Y, Tiftikci A, et al. IgG-based elimination diet in migraine plus irritable bowel syndrome. Headache, 2013,53(3):514-525.

39. Srinivasan V, Lauterbach E C, Ho K Y, et al. Melatonin in antinociception: its therapeutic applications. Curr Neuropharmacol, 2012,10(2):167-178.

40. Lu W Z, Gwee K A, Moochhalla S, et al. Melatonin improves bowel symptoms in female patients with irritable bowel syndrome: a double-blind placebo-controlled study. Aliment Pharmacol Ther, 2005,22(10):927-934.

41. Tabeeva G R, Sergeev A V, Gromova S A. Possibilities of preventive treatment of migraine with the MT1- and MT2 agonist and 5-HT2c receptor antagonist agomelatin (valdoxan). Zh Nevrol Psikhiatr Im S S Korsakova, 2011,111(9):32-36.

42. Duffy R A. Potential therapeutic targets for neurokinin-1 receptor antagonists. Expert Opin Emerg Drugs, 2004,9(1):9-21.

\section{Figures}




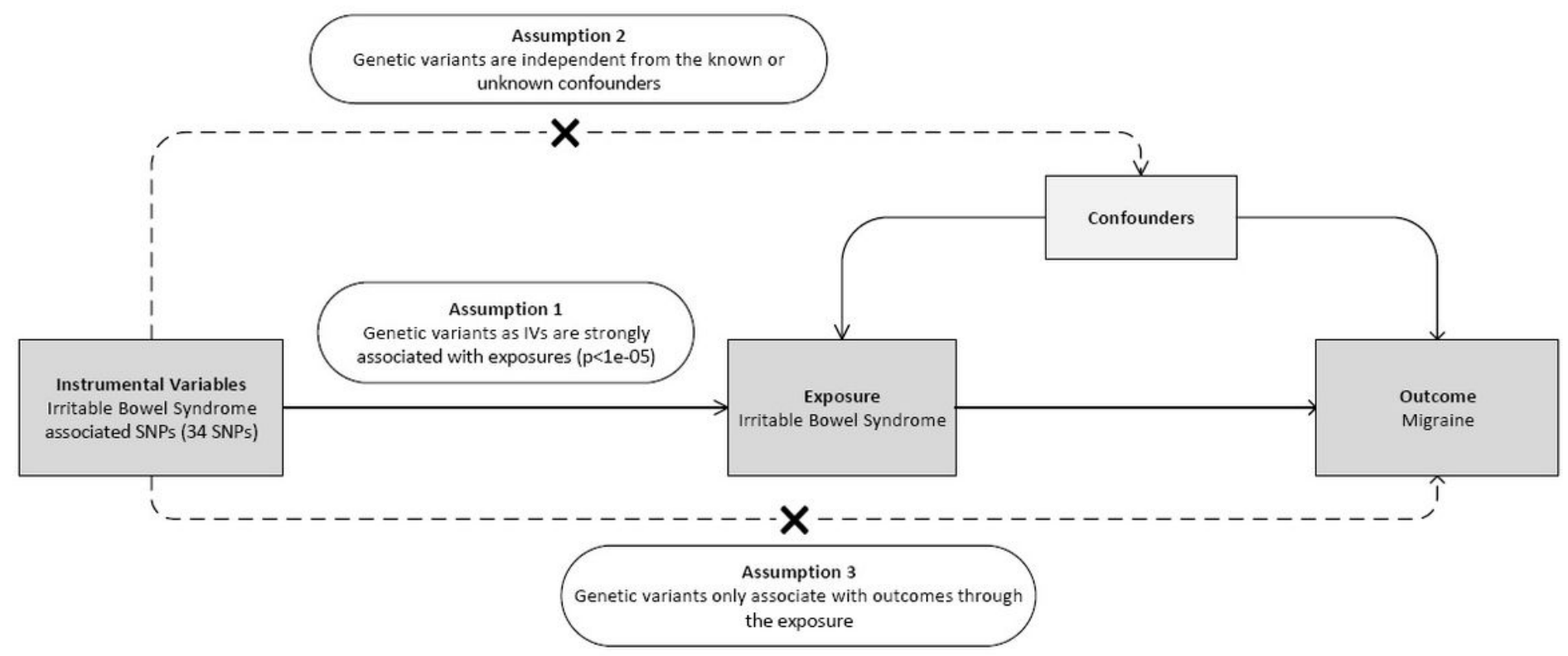

\section{Figure 1}

Diagram of the Mendelian Randomization Assumptions Supporting a Two-sample Mendelian Randomization Analysis of the Causal Effect of Irritable Bowel Syndrome on Migraine.

\section{Method}

Inverse variance weighted

MR Egger

Maximum likelihood

Weighted median

Robust adjusted profile score (RAPS)
nSNP Odds Ratio(95\% Cl)

$34 \quad 1.09(1.01-1.17)$

34

$1.14(1.00-1.30)$

34

1.09 ( 1.01-1.18)

34

$1.10(0.99-1.22)$

34

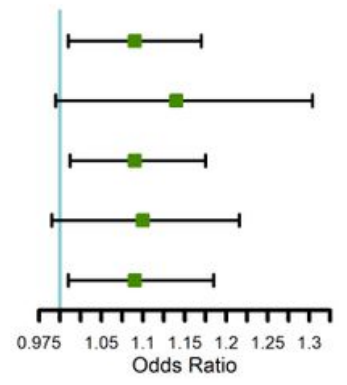

p-value

0.03

0.07

0.02

0.08

0.03

\section{Figure 2}

MR Estimates from Each Method of Assessing the Causal Effects of Irritable Bowel Syndrome on Migraine. 


\section{MR Test}

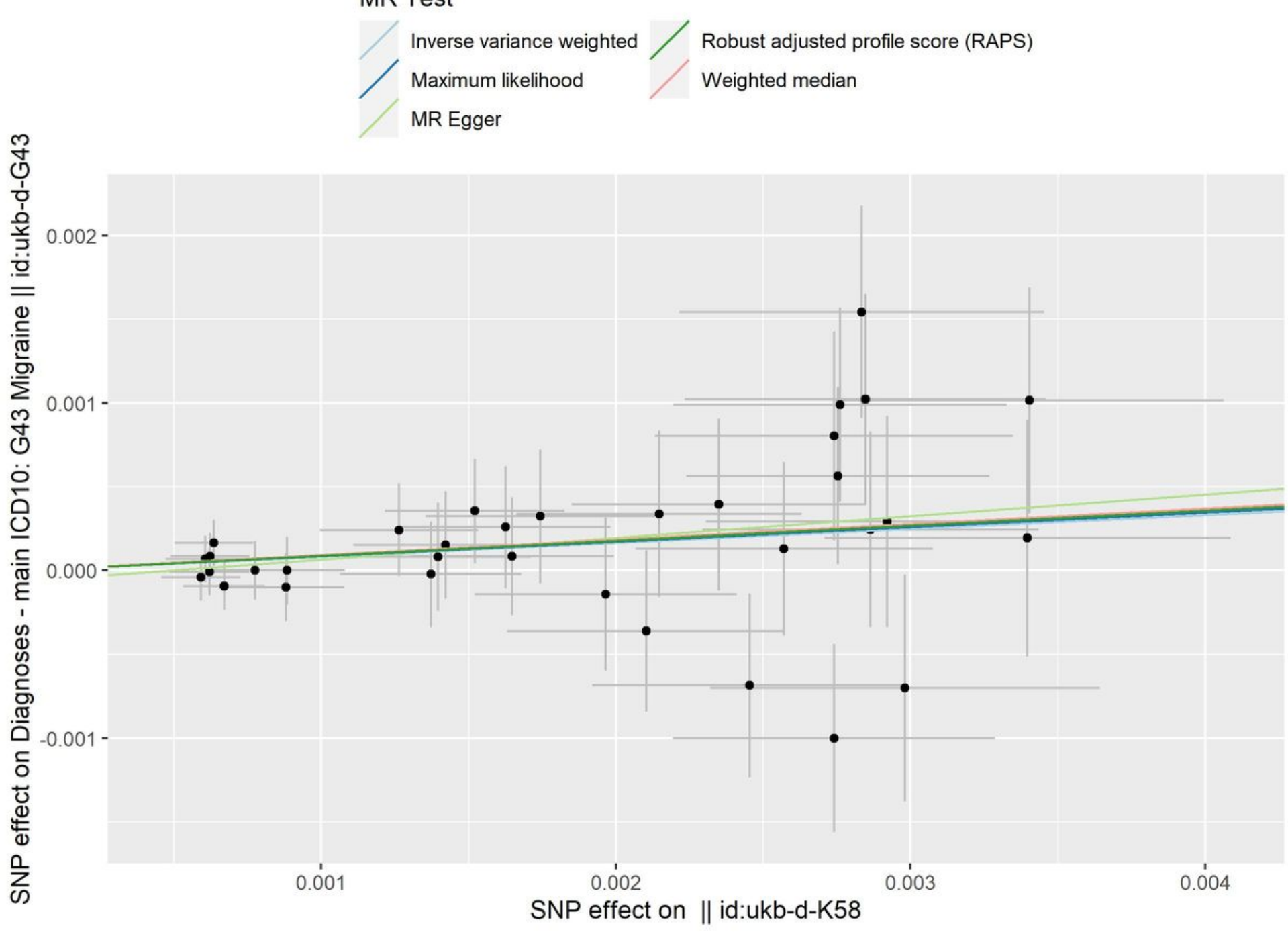

\section{Figure 3}

Scatter plots of the genetic causal associations with Irritable Bowel Syndrome against Migraine using different MR methods.

\section{Supplementary Files}

This is a list of supplementary files associated with this preprint. Click to download.

- SupplementaryFiguresandTables.docx 